\title{
Etude expérimentale de la dénitrification in situ en eaux courantes : application à la rivière Charente
}

\author{
M. Torrel \\ J.-P. Rebillard1 \\ H. Ayphassorhol \\ L. Labroue ${ }^{2}$ \\ C. Helmer ${ }^{2}$
}

Mots clés : dénitrification, activité bactérienne, sédiment, nitrates, rivière, méthodologie.

L'expérimentation, menée au cours d'un cycle annuel sur deux secteurs différents de la rivière Charente à l'aval d'Angoulème, a pour objectif de quantifier le taux de dénitrification pour les besoins d'un modèle hydrologique nitrates.

La méthode de blocage du stade final de la réaction de dénitrification à l'acétylène a permis le dosage du protoxyde d'azote à partir de cloches à sédiment et d'un conteneur immergé en pleine eau.

Les résultats montrent que, si ce phénomène n'a pas lieu dans la tranche d'eau, la vitesse de dénitrification à l'interface eau-sédiment, facteur $\mathrm{K}$ exprimé en $\mathrm{mg}$ de nitrates dénitrifiés par $\mathrm{m}^{2}$ par jour, peut être considérée conme constante et indépendante des variations en température, en teneurs en nitrates et oxygene dissous de l'interface.

Sur un cycle annuel, en l'absence d'influence marquée des paramètres environnementaux, le taux moyen de dénitrification dans la rivière Charente est de $682 \mathrm{mg}$ de $\mathrm{NO}_{3}-/ \mathrm{m}^{2} / \mathrm{j}$.

La comparaison des mesures obtenues par cette méthode à celles évaluées par le calcul (bilan azoté) montre qu'il est préférable d'estimer le taux de dénitrification par dosage direct plutôt qu'à partir de l'analyse des formes de l'azote (disparition du $\mathrm{NO}_{3}^{-}$ou défaut du bilan).

\section{In situ assessment of denitrification in running waters : example of the Chanente river}

Keywords : denitrification, bacterial activity, sediment, nitrate, river, methodology

The experiment lasted one year at two stations of the Charente river, downstream from Angoulème. The aim of this study was to quantify the denitrification rate and to insert it in a nitrate hydrological model. We used four benthic chambers located on the sediment and a submerged 20 litre bottle in the water.

The nitrous oxide determination, after acetylene inhibition of its reduction, was made by GPC. We observed no $\mathrm{N}_{2} \mathrm{O}$ production in the water. The denitrification rate, expressed as mg of nitrates. $M^{-2} \cdot d^{-1}$, can be considered as a constant and is independent of the temperature variation, nitrate and dissolved oxygen concentrations of the sediment-water system.

On a annual cycle, whithout the marked influence of environmental factors, the mean rate of denitrification in the river Charente is about $682 \mathrm{mg} \cdot \mathrm{m}^{-2} \cdot \mathrm{d}^{-1}$.

The comparison of the field measurements and evaluation by calculation of the denitrification phenomenon shows that it is preferable to estimate the denitrification rate by direct titration instead of nitrogen form analysis.

\section{Introduction}

Le développement des différents secteurs économiques utilisateurs concurrentiels d'eau, notamment l'industrie, la distribution publique d'eau potable,

1. Cemagref, Groupement de Bordeaux, Division Qualité des Eaux, F-33610 Gazinet.

2. Laboratoire d'Hydrobiologie, URA 695 CNRS, Université Paul Sabatier, 118, route de Narbonne, F-31062 Toulouse Cedex. le tourisme, l'agriculture, a considérablement compliqué la gestion des eaux continentales. Dans le cas de la rivière Charente, cette difficulté est encore plus sensible puisque l'aménagement des eaux continentales a un impact direct sur le milieu marin et les activités qui lui sont associées, principalement la conchyliculture. Drainant un bassin versant d'une superficie voisine de $10000 \mathrm{~km}^{2}$, la Charente reçoit, outre la fraction non épurée des rejets urbains et industriels, les pollutions diffuses liées aux pratiques agricoles intensives. 
C'est ainsi que, dans les vingt dernières années, les concentrations en nitrates y ont plus que doublé.

Afin d'évaluer les risques de compétitions entre activités utilisatrices d'eau et les atteintes à l'environnement, un programme d'étude financé par le Ministère de la Recherche a été lancé pour établir un outil rationnel de gestion de la ressource en eau. Compte tenu de l'importance de l'azote dans les processus d'eutrophisation, l'établissement d'un modèle numérique de transfert des nitrates (Ma 1991) est apparu essentiel pour une simulation et une précision des flux.

Au sein du cycle de l'azote, intervient un processus qui est à considérer avec attention puisqu'il conduit à une perte nette d'azote pour le milieu aquatique : il s'agit de la dénitrification au niveau de l'interface eau/sédiment et, dans des conditions particulières, dans la masse d'eau. La réduction des nitrates est assurée, en anaérobiose, par des populations bactériennes très variées grâce à deux mécanismes dissimilatoires : réduction en $\mathrm{NH}_{4}{ }^{+}$par des microorganismes anaérobies ou facultatifs et dénitrification par des germes aérobies capables de se développer en anaérobiose en présence de nitrates ou de nitrites (Painter 1977, Gorlenko et al. 1983 , Tiedje 1988).

La dénitrification dépend principalement de la concentration en $\mathrm{O}_{2}$ dissous et moins strictement de la teneur en nitrates et en matière organique facilement dégradable et de la température (Knowles 1982, Seitzinger 1988). Les deux systèmes enzymatiques qui régissent la dénitrification réagissent différemment au taux d'oxygène dissous : la synthèse des nitrates-réductases ( $\mathrm{NaR})$ est déclenchée par une chute régulière de la teneur en $\mathrm{O}_{2}$ dissous ou en conditions semi-anaérobies. Par contre, celle des nitrites-réductases (NiR) est fortement réprimée par la présence d' $\mathrm{O}_{2}$. Dans les eaux douces, la réaction nécessite une concentration en $\mathrm{O}_{2}$ dissous inférieure à $0,2 \mathrm{mg} / \mathrm{l}$ et se trouve encore efficace pour des concentrations en $\mathrm{NO}_{3}-$ faibles $\left(10 \mu \mathrm{g} \mathrm{N}-\mathrm{NO}_{3}-/ \mathrm{l}\right)$. Cependant, on constate que jusqu'à $2 \mathrm{mg} \mathrm{d} \mathrm{O}_{2} / 1$ d'eau, l'activité dénitrifiante n'est pas inhibée mais seulement réduite et qu'elle se poursuit même à des concentrations de 6 à $8 \mathrm{mg} \mathrm{d} \mathrm{d}_{2} / \mathrm{l}$.

L'interface eau-sédiment représente une zone d'échanges très active. Dans les eaux oxygénées, c'est le taux de diffusion des nitrates (nitrification et apports de la colonne d'eau) dans l'épaisseur des sédiments qui détermine l'activité dénitrifiante. Au niveau des sites de production des nitrates, le déficit local en $\mathrm{O}_{2}$ dissous entraine la mobilitê de l'interface aéro-anaérobie (Tiedje 1982). En conditions «normales », la dénitrification dans la colonne d'eau est peu active, voire inexistante.

La quantification de cette réaction de dénitrification au niveau de la Charente est indispensable au bon fonctionnement du modèle « nitrates ». Elle permet de s'affranchir des valeurs tirées de la littérature (Toms 1975, Whitehead 1984) et généralement admises sans vérification. La grande majorité des travaux réalisés dans ce domaine met en œuvre des techniques issues de la microbiologie des sols et concerne des expérimentations soit en laboratoire, soit in situ, principalement en eaux douces stagnantes (lacs, gravières) et en milieu marin.

Nous décrirons ici une expérimentation menée in situ en eau courante à l'aide de cloches à sédiment.

\section{Matériel et méthode}

\subsection{Sites d'intervention}

L'étude de la dénitrification a été conduite durant un cycle annuel sur la rivière Charente à l'aval de l'agglomération d'Angoulème (région PoitouCharente, centre ouest de la France). Deux sites expérimentaux ont été retenus :

- le bief de Nersac, caractérisé par une tranche d'eau moyenne de 3 à $4 \mathrm{~m}$ et un fonds de graviers et cailloux associés à des trous vaseux. La colonisation par les macrophytes y est importante d'avril à novembre et un floc mobile de couleur brune tapisse le sédiment.

- le bief de Juac, caractérisé par une tranche d'eau comprise entre 1,5 et $2 \mathrm{~m}$ et un sédiment brunnoir, sableux, avec des débris organiques. Le courant y est faible et les macrophytes peu abondants du fait d'un couvert végétal rivulaire important.

La présente communication fait état des résultats obtenus sur neuf campagnes de mesures effectuées de mai 1991 à avril 1992 sur les sédiments et en pleine eau.

\subsection{Matériel utilisé}

Quatre cloches benthiques ont été réalisées dans du tube PVC de $30 \mathrm{~cm}$ de diamètre $\left(730 \mathrm{~cm}^{2}\right.$ de surface couverte) d'après le modèle utilisé en gravières par Labroue et al. (1988). 
Deux tubulures apicales permettent, grâce à une pompe péristaltique située en surface, l'homogénéisation de la masse d'eau contenue dans chaque cloche (15 litres) ainsi que toutes les opérations d'injection et de prélèvement. Une des cloches comporte un orifice à joint torique permettant l'introduction d'une sonde oxymétrique.

Sur chaque site, les quatre cloches à sédiment ont été immergées et disposées sur le fond selon un carré d'une dizaine de mètres de côté par un plongeur. L'utilisation de ces quatre systèmes permet la prise en compte de la variabilité des mesures et de l'hétérogénéité des sédiments. Un conteneur de 201 d'eau de rivière immergé à mi-profondeur a servi à la mesure de la dénitrification en pleine eau.

\subsection{Techniques analytiques}

Nous avons appliqué la méthode de dosage du protoxyde d'azote $\left(\mathrm{N}_{2} \mathrm{O}\right)$ après blocage du stade final de la réaction de dénitrification par l'acétylène. Cette méthode rend compte d'une quantité d'enzymes actives plutôt que d'une densité de population bactérienne. C'est une mesure de l'activité d'un ensemble de microorganismes différents possédant une fonction en commun.

Une fois le matériel mis en place sur le fond et le raccordement des tuyaux souples effectué avec la surface, $1500 \mathrm{ml}$ de $\mathrm{C}_{2} \mathrm{H}_{2}$ sont injectés dans chaque enceinte ( $10 \%$ du volume). Après $10 \mathrm{mn}$ de brassage par circulation d'eau à l'aide de la pompe de surface, ce qui assure la diffusion de $\mathrm{C}_{2} \mathrm{H}_{2}$ dans la couche superficielle de sédiment ou dans la masse d'eau, un premier prélèvement est effectué au temps $\mathrm{t}_{0}(6.5 \mathrm{ml}$ de liquide homogénéisé sont aspirés à la seringue et réinjectés dans des cubes à vides Vénoject de $13 \mathrm{ml}$ de volume total) et cinq prélèvements horaires successifs sont réalisés de la même manière ( $t_{1}$ à $\left.t_{5}\right)$. Les tubes d'échantillons sont ensuite stockés en glacières en vue d'analyse. Un protocole identique a été appliqué au conteneur de $20 \mathrm{l}$.

L'évolution de la physicochimie des eaux des enceintes est suivie par enregistrements en continu de la consommation en $\mathrm{O}_{2}$ dissous et de la température et par prélèvements aux temps $t_{0}$ et $t_{5}$ d'échantillons destinés à l'analyse systématique des nitrates. Pour certaines campagnes, l'analyse complémentaire des $\mathrm{NO}_{2}{ }^{-}, \mathrm{NH}_{4}{ }^{+}$et azote total Kjeldahl a été réalisée.

Pour le dosage du $\mathrm{N}_{2} \mathrm{O}$ en laboratoire, les prélèvements en tubes Vénoject, conservés jusqu'à 7 jours sans modification, sont mis à température ambiante. Ces tubes, encore sous léger vide, sont percés pour être mis en équilibre avec l'atmosphère. Après agitation, $200 \mu \mathrm{l}$ de gaz sont prélevés et injectés dans un chromatographe en phase gazeuse, détecteur à capture d'électrons (Delsi Série 30). Cet appareil est équipé d'un système back-flush qui évite d'envoyer l'acétylène sur le détecteur et d'une alimentation pulsée variable selon la concentration (entre 0,3 et 150 ppm). Le gaz vecteur utilisé est un mélange argonméthane (95/5) améliorant la sensibilité. L'obtention de pics hauts et étroits autorise la seule prise en compte de leur hauteur sans intégration des surfaces sous la courbe. Un étalonnage préalable à l'air $(0,3 \mathrm{ppm})$ et par gaz étalon ( 25,50 et $100 \mathrm{ppm})$ permet d'obtenir directement les valeurs xi en ppm de $\mathrm{N}_{2} \mathrm{O}$ de la phase gazeuse de l'échantillon.

Connaissant le coefficient de solubilité de $\mathrm{N}_{2} \mathrm{O}$ dans l'eau, on évalue par simple calcul la concentration initiale totale en $\mathrm{N}_{2} \mathrm{O}$ de chaque échantillon (Tableau 1). Pour chacune des cloches, la courbe des concentrations en $\mathrm{N}_{2} \mathrm{O}$ en fonction du temps traduit la cinétique de la réaction. En éliminant le début de courbe qui correspond au temps nécessaire à la diffusion de $\mathrm{C}_{2} \mathrm{H}_{2}$ et la fin de courbe qui traduit une modification des réactions dans les cloches $\left(\mathrm{C}_{2} \mathrm{H}_{2}\right.$ transformé, effet de confinement bien marqué au bout de 6 heures), nous retenons la pente moyenne de la courbe et donc deux concentrations $x$ de $\mathrm{N}_{2} \mathrm{O}$ en fonction du temps. Cela permet d'exprimer une production moyenne de $\mathrm{N}_{2} \mathrm{O}$ en $\mu \mathrm{g} / \mathrm{m}^{2} / \mathrm{h}$ qui sera égale à ( $\Delta \mathrm{x} *$ volume enceinte) $/$ (surface enceinte * temps), avec $\Delta \mathrm{x}$ en $\mathrm{ppm}(\mu \mathrm{g} / 1$ d'eau), volume de l'enceinte en litre, surface de l'enceinte en $\mathrm{m}^{2}$ et temps en heure.

\section{Résultats et discussion}

\subsection{Production de protoxyde d'azote dans les enceintes}

Pour chacun des deux sites et pour chacune des neuf expérimentations, nous disposons de quatre valeurs de production moyenne de $\mathrm{N}_{2} \mathrm{O}$ exprimées en $\mu \mathrm{g} / \mathrm{m}^{2} / \mathrm{h}$ ainsi que d'une série d'analyses de paramètres physicochimiques de l'eau en fonction du temps d'expérimentation (formes de l'azote, $\mathrm{O}_{2}$ dissous, température). La production moyenne de $\mathrm{N}_{2} \mathrm{O}$ est calculée par moyenne arithmétique des quatre valeurs issues des mesures de terrain. Le tableau 2 présente cette production moyenne et son équivalence en quantité de nitrates dénitrifiés pour chacun des sites. 
Tableau 1. Mode de calcul de la concentration initiale en $\mathrm{N}_{2} \mathrm{O}$ dans l'eau.

Table 1. Calculation method of $\mathrm{N}_{2} \mathrm{O}$ initial concentration in water.

La quantité totale de protoxyde d'azote présente sous forme gazeuse dans un tube vénoject sera la somme de la fraction déjà présente dans le vénoject (à sa mise sous vide), de la fraction provenant de l'échantilton (Gi) et de la fraction inırođuite à l'équilibration avec l'atmosphère lors de la mesure.

soit $x \mathrm{i}=$ quantité de $\mathrm{N}_{2} \mathrm{O}$ en $\mathrm{ppm}$ volume $(\mu \mathrm{l} / \Omega)$ de la fraction gazeuse de l'échantillon

La constante d'equilibre de $\mathrm{N}_{2} \mathrm{O}$ entre phase gazeuse et phase aqueuse est :

$\mathrm{K}_{0}=2,7.10^{-2}$ mole. $\mathrm{l}^{-1} \cdot \mathrm{atm}^{-1}$ à $22^{\circ} \mathrm{C}$.

1 mole $\mathrm{N}_{2} \mathrm{O}=44 \mathrm{~g}$ pour $22,4 \mathrm{l}$ soil une densité de 1,964 . Dans l'eau, nous aurons $44 * \mathrm{~K}_{0}=1,188$

Avec une constante de répartition eau-air $=1,188 / 1,964=0,6$ (rapport en masse), la teneur globale en $\mathrm{N}_{2} \mathrm{O}$ (phase gazeuse Gi et phase aqueuse $\mathrm{Li}$ ) de l'échantillon est :

$(\mathrm{xi} * 1,964 *$ Vair en $m l)+(\mathrm{xi} * 1,964 * 0,6 *$ Veau en $m l)-(\text { Vair en } m l * 1,964 * 0.3)^{\mathrm{a}}-(0,470 * \text { Vuénoject en ml })^{\mathrm{b}}$

a : $\mathrm{N}_{2} \mathrm{O}$ en $\mu \mathrm{g}$ introduit par équilibration dans le vénoject soit $x \cdot 10^{-3} \mu \mathrm{g} /$

$\mathrm{b}: \mathrm{N}_{2} \mathrm{O}$ en $\mu \mathrm{g}$ présent dans un vénoject de $13 \mathrm{ml}$ soit $\mathrm{y} \cdot 10^{-3} \mu \mathrm{g} / \mathrm{l}$

Les Vénojects utilisés ont un volume de $13 \mathrm{ml}$ soit $6,5 \mathrm{ml}$ air et $6,5 \mathrm{ml}$ eau (échantillon prélevé).

La teneur initiale en $\mathrm{N}_{2} \mathrm{O}$ dans l'eau exprimée en $\mu \mathrm{g} / \mathrm{I}$ est :

$x=[(x i * 12,77)+(x i * 7,66)-3,83-6,11] / 6.5$ soit $x=3,14 x i-1,53$

Tableau 2. Taux horaire moyen de dénitrification exprimé en $\mathrm{N}_{2} \mathrm{O}$ produit et en $\mathrm{NO}_{3}$ - dénitrifié.

Table 2. Hourly mean denitrification rate expressed in $\mathrm{N}_{2} \mathrm{O}$ produced and $\mathrm{NO}_{3}-$ denitrificated.

\begin{tabular}{|c|c|c|c|c|c|c|c|c|c|}
\hline & 5/1991 & $6 / 1991$ & $7 / 1991$ & $8: 1991$ & $9 / 1991$ & $10 / 1991$ & $11 / 1991$ & $3 / 1992$ & $4 / 1992$ \\
\hline $\begin{array}{c}\text { Nersac } \\
\mu \mathrm{mol} \text { N2O/m } 2 / \mathrm{h}\end{array}$ & 122 & 186 & 333 & 169 & 219 & 166 & 238 & 175 & 156 \\
\hline $\begin{array}{c}\text { mg NO3 } / m_{2} / m \\
\text { Juac }\end{array}$ & 15,1 & 23,1 & 41,3 & 20,9 & 27,2 & 20,6 & 29,5 & 21,7 & 19,4 \\
\hline$\mu \mathrm{mol} \mathrm{N} 2 \mathrm{O} / \mathrm{m} 2 \mathrm{~h}$ & 834 & 610 & 284 & 80 & 118 & 79 & 37 & 123 & 198 \\
\hline $\mathrm{mg} \mathrm{NO3} \mathrm{mm} 2 \mathrm{~h}$ & 103,4 & 75,6 & 35,2 & 9,9 & 14,6 & 9,8 & 4,6 & 15,2 & 24,6 \\
\hline
\end{tabular}

Une analyse de variance regroupe les 18 valeurs obtenues sur les neuf campagnes et les deux sites en une même population et n'indique aucune différence significative au seuil de $1 \%$ entre les types de sédiments et entre les variations dans le temps (Tableau 3 ).

Les nitrates jouent le rôle d'inducteur de réductase, le taux de dénitrification devrait être corrélé positivement à leur concentration, surtout si celleci est faible (faibles $\mathrm{Km}$ des réductases). Aucune liaison évidente n'est établie ici entre les données

Tableau 3. Analyse statistique des données de dénitrification.

Table 3. Statistical analysis of denitrification data.

\begin{tabular}{crcrc}
\hline $\begin{array}{c}\text { Source de } \\
\text { variation }\end{array}$ & Dispersion & $\begin{array}{c}\text { Degré de } \\
\text { liberté }\end{array}$ & Variance & F \\
\cline { 1 - 2 } Factorielle & & & & \\
- Espace & 50736022 & 1 & 50736022 & 0.72 \\
- Temps & 567566788 & 8 & 70945848 & 0.81 \\
Résiduelle & 699718644 & 8 & 87464830 & \\
\hline
\end{tabular}

physicochimiques $\left(\mathrm{NO}_{3}{ }^{-}, \mathrm{O}_{2}\right.$, température) et la production de $\mathrm{N}_{2} \mathrm{O}$ dans le temps.

Ainsi, dans les conditions de l'expérience, cette production serait donc peu ou pas dépendante de la nature des sédiments et de l'évolution des paramètres physico-chimiques des eaux.

Les figures 1 et 2 représentent les cinétiques d'évolution des nitrates : les nitrates disparus sont soit dénitrifiés et donc perdus pour le système, soit assimilés et donc recyclés dans le système. Pour le site de Nersac, le taux moyen de dénitrification (facteur $\mathrm{K})$ est de $24 \mathrm{mg}$ de $\mathrm{NO}_{3}-$ dénitrifié $/ \mathrm{m}^{2} / \mathrm{h}$. Pour celui de Juac, il est de $32 \mathrm{mg}$ (les deux premières valeurs semblent surestimées du fait d'un positionnement probablement trop profond des cloches dans le sédiment). Ces valeurs variant dans une gamme réduite (à l'intérieur d'une unité logarithmique), la valeur globale moyenne retenue sera donc la moyenne de ces deux taux ( $\mathrm{cf} \S 2.3$ ). 
Fig. 1. Evolution du taux de disparition des nitrates et de l'activité dénitrifiante à la station de Nersac sur un cycle annuel.

Fig. 1. Evolution of nitrate rate removal and denitrifying activity during a one year cycle at Nersac station.

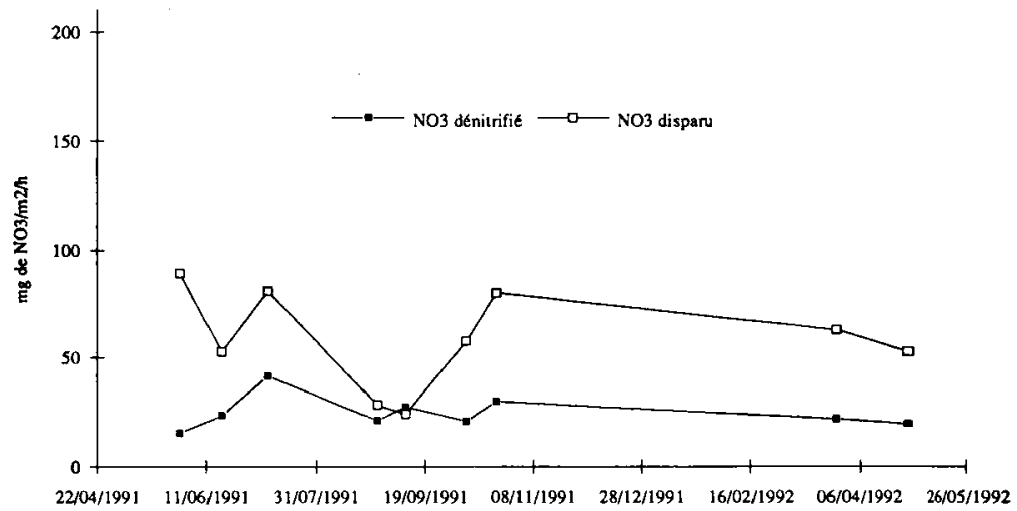

Fig. 2. Evolution du taux de disparition des nitrates et de l'activité dénitrifiante à la station de Iuac sur un cycle annuel.

Fig. 2. Evolution of nitrate rate removal and denitrifying activity during a one year cycle at Juac station.

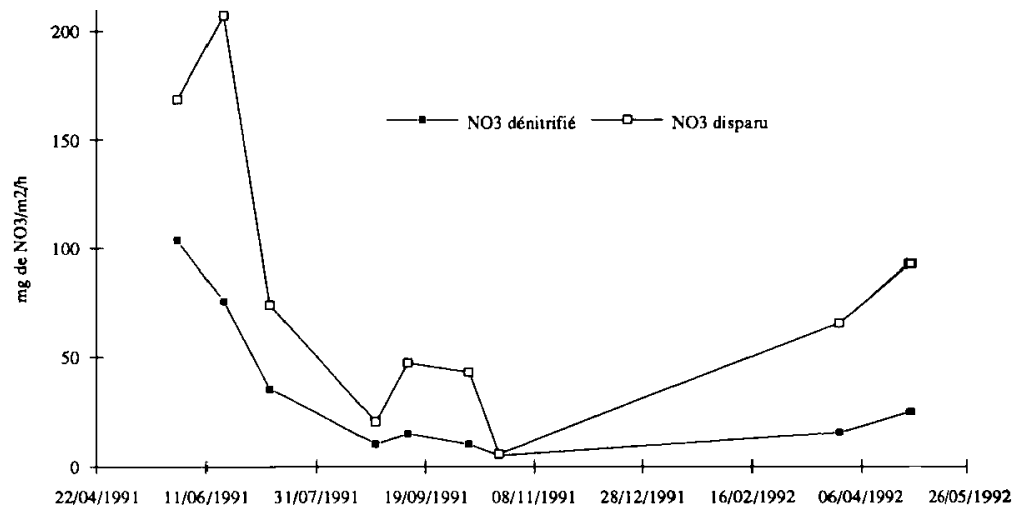


Nous avons pu vérifier l'absence d'accumulation de $\mathrm{N}_{2} \mathrm{O}$ dans l'eau des enceintes au cours de trois manipulations sans injection d'acétylène : les nitrates à une concentration inférieure à $25 \mathrm{mg} / \mathrm{l}$ d'eau n'inhibent pas la réduction du $\mathrm{N}_{2} \mathrm{O}$. Aux fortes concentrations en $\mathrm{NO}_{3}-$, la diffusion des nitrates vers les microsites anaérobies augmente. Il y a alors inhibition de la réduction du NO et donc accumulation de $\mathrm{NO}_{2}-$ et par là même inhibition de la réduction de $\mathrm{N}_{2} \mathrm{O}$ de sorte que le rapport $\mathrm{N}_{2} \mathrm{O} / \mathrm{N}_{2}$ augmente (Labroue et al. 1991).

Nous constatons aussi l'absence de production de $\mathrm{N}_{2} \mathrm{O}$ dans la colonne d'eau : les prélèvements réalisés montrent une teneur en $\mathrm{N}_{2} \mathrm{O}$ constante pendant 6 heures et identique à celles des $t_{0}$ correspondant aux cloches benthiques.

\subsection{Evolution des formes de l'azote}

Dans ces eaux en général bien oxygénées et en l'absence d'inhibition par l'acétylène, les nitrates sont formés dans les microzones oxydées du sédiment et proviennent de l'oxydation de l'ammoniaque relargué in situ et par les zones anaérobies inférieures. Selon le potentiel d'oxydoréduction de l'interface, les populations bactériennes interviennent d'une manière différente dans les réactions conduisant à la disparition des nitrates (réduction ou dénitrification). Lorsque la dénitrification est effective, c'est la source de $\mathrm{NO}_{3}$ - immédiatement disponible qui sera utilisée (nitrates produits dans l'interface ou nitrates diffusant depuis la colonne d'eau).

En règle générale, l'activité dénitrifiante mesurée est souvent inférieure à l'activité réelle car l'utilisation de $\mathrm{C}_{2} \mathrm{H}_{2}$ inhibe la nitrification. De plus, dans le cas d'une diffusion partielle de l'acétylène dans le sédiment, le blocage de la réduction de $\mathrm{N}_{2} \mathrm{O}$ est limité. Il en est ainsi lorsque la zone de dénitrification est éloignée de la surface du sédiment ou lorsque, pour de faibles concentrations en nitrates dans les eaux et les sédiments, les réactions nitrificationdénitrification sont couplées.

Dans notre expérimentation, la chute régulière mais faible de la teneur en $\mathrm{O}_{2}$ dissous dans les enceintes ne semble stimuler que modérément l'activité dénitrifiante. Par contre, l'action de $\mathrm{C}_{2} \mathrm{H}_{2}$ sur la nitrification limite les sources de $\mathrm{NO}_{3}$ - disponibles. La colonne d'eau représente la principale source de nitrates si le taux de dénitrification reste du même ordre de grandeur que le taux de disparition des nitrates dans l'eau.

Le tableau 4 montre que, comme prévu, la mesure directe (production de protoxyde d'azote par unité de temps) est inférieure au taux de disparition des nitrates de l'eau. Cela s'explique par une disparition d'une partie de ces nitrates probablement due à la consommation (assimilation) et à la réduction non assimilative en $\mathrm{NH}_{4}{ }^{+}$. Nous observons bien une légère augmentation de l'azote ammoniacal en cinq heures. Le reste (en moyenne $36 \%$ des nitrates disparus du système) est dénitrifié.

L'estimation de la dénitrication par la méthode des bilans présuppose que la masse d'eau dans les cloches benthiques est un système isolé (en particulier, pas d'échanges eau-sédiment). Le bilan se fera par rapport aux dosages des formes d'azote $\mathrm{NO}_{3}{ }^{-}$, $\mathrm{NO}_{2}{ }^{-}, \mathrm{NH}_{4}{ }^{+}, \mathrm{NK}$ ). Les taux d'évolution horaire des formes de l'azote dans les cloches ne permettent pas d'équilibrer une balance des formes azotées. Nous avons ramené ces valeurs en pourcentage de l'azote total et calculé les valeurs de $\mathrm{N}-\mathrm{N}_{2} \mathrm{O}$ théoriques nécessaires pour avoir un système conservatif.

Tableau 4. Consommation de nitrates et production de $\mathrm{N}_{2} \mathrm{O}$ dans les enceintes.

Table 4. Nitrate consumption and $\mathrm{N}_{2} \mathrm{O}$ production in benthic chambers.

\begin{tabular}{|c|c|c|c|c|c|}
\hline & $7 / 1991$ & $8: 1991$ & $9 / 1991$ & $10 \mid 1991$ & $11 / 1991$ \\
\hline \multicolumn{6}{|l|}{ Nersac } \\
\hline N-NO3 $\mu \mathrm{gIlh}$ & 91 & 29 & 30 & 63 & 88 \\
\hline $\mathrm{N}-\mathrm{N} 2 \mathrm{O} \mu \mathrm{g} / \mathrm{h}$ & 45 & 23 & 30 & 23 & 32 \\
\hline \multicolumn{6}{|l|}{ Juac } \\
\hline N-NO3 $\mu \mathrm{gllh}$ & 93 & 23 & 56 & 47 & 6 \\
\hline $\mathrm{N}-\mathrm{N} 20 \mathrm{\mu g} / \mathrm{hh}$ & 39 & 11 & 16 & 11 & 5 \\
\hline
\end{tabular}


Dans le tableau 5, la comparaison des taux horajres de protoxyde d'azote mesurés (données expérimentales) et calculés (défaut de bilan) montre que, si les 2 séries de valeurs sont voisines, les taux issus des mesures de terrain sont plus faibles.

L'azote total devrait être conservé entre $t_{0}$ et $t_{5}$ puisqu'on suppose que rien ne s'échappe du système. Les différences observées (avec $\Sigma N$ à $t_{5}<\Sigma N$ à $t_{0}$ ) ont pour cause probable les erreurs de dosage sur l'azote organique qui est de plus difficile à doser. Les précisions des dosages sont de $\pm 0,1 \mathrm{mg} / 1$ pour $\mathrm{NO}_{3}{ }^{-}$et pour $\mathrm{N}$ organique. Les variations en concentrations sont très faibles sur cinq heures d'expérimentation et les erreurs sur les dosages s'additionnant, les calculs des taux horaires seront entachés d'une erreur d'autant plus importante que ces variations entre $t_{0}$ et $t_{5}$ seront plus faibles. Ainsi, une variation de $-4,59 \%$ par heure d'azote organique correspondra à une dénitrification calculée énorme.

Un mauvais positionnement de l'enceinte peut entrainer une remise en suspension des sédiments : c'est le cas à Juac en août 1991 où l'on observe une augmentation de l'azote organique et ammoniacal d'où une perte apparente (consommation) de $\mathrm{N}_{2} \mathrm{O}$ très importante $(-5,87 \%)$ tout à fait ir réaliste. Dans ce cas, les échantillons d'eau étaient anormalement chargés de matières en suspension.

La méthode des bilans semble donc peu fiable et incapable de rendre compte de l'activité dénitrifiante. N'ayant accès qu'à la masse d'eau des cloches, on ignore ce qui se passe dans les sédiments. Sur l'ensemble des échanges entre l'eau, l'interface et le sédiment actif, les réactions biochimiques de faible intensité se déroulant sur un temps très court ne sont pas traduites. Cela s'ajoute au déficit inhérent au principe même de la mesure (confinement dans les enceintes et l'effet de l'acétylène).

En revanche, la mesure directe, elle, ne fait intervenir qu'un seul paramètre $\left(\mathrm{N}_{2} \mathrm{O}\right)$.

\subsection{Taux de disparition des nitrates $K$}

L'évaluation d'un taux de disparition des nitrates $\mathrm{K}$ conduit à formuler des hypothèses simplificatrices (déjà précédemment admises) : la vitesse de dénitrification à l'interface eau/sédiment est constante par unité de surface et ne dépend ni de la concentration en $\mathrm{NO}_{3}-$ de l'eau, ni de la température, ni de la nature plus ou moins organique du sédiment. La dénitrification dans la masse d'eau est toujours nulle.

Pour être incorporée dans le modèle général " $\mathrm{NO}_{3}-$ Charente", cette information doit être exprimée sous la forme d'une variation de la concentration en nitrates dans l'eau en fonction de la constante « taux de dénitrification ».

Pour ce faire, nous considérons une tranche d'eau élémentaire verticale perpendiculaire à l'axe de l'écoulement 1, de hauteur p (profondeur d'eau), de largeur $1 \mathrm{~m}$ et d'épaisseur dl (en $\mathrm{m}$ ). La tranche d'eau sera supposée se déplacer avec une vitesse uniforme $\mathrm{dl} / \mathrm{dt}$ et rester donc au-dessus de la surface de sédiment ds ( $\mathrm{ds}=\mathrm{dl} * 1 \mathrm{~m}$ ) pendant le temps dt.

A $t=0$, la concentration en $\mathrm{NO}_{3}{ }^{-}$de la tranche d'eau est appelée $\mathrm{C}(\mathrm{mg} / \mathrm{l})$

A $t=d t$, la concentration en $\mathrm{NO}_{3}{ }^{-}$de la tranche d'eau est $C+d C(m g / 1)$

Tableau 5. Evolution du ratio formes azotées/azote total dans les enceintes exprimé en $\%$ par heure.

Table. 5. Evolution of the ratio nitrogen compounds/total nitrogen in benthic chambers (in $\%, h^{-1}$ ).

\begin{tabular}{|c|c|c|c|c|c|}
\hline $\operatorname{Nersac}(\mathrm{en} \phi(\mathrm{h})$ & 7I5) & 8159 & 91591 & 101991 & $11 / 1991$ \\
\hline$\Delta \mathrm{N}(\mathrm{NO} 3+\mathrm{NO} 2+\mathrm{NH}) / \mathrm{Nt}^{\circ}$ & $-3,03$ & $-0,36$ & $-0,56$ & $-1,14$ & $-1,49$ \\
\hline$\Delta \mathrm{N}$ organique $\mathbf{N} \mathbf{N}$ & 0 & 4.59 & 0 & $-0,9$ & $-1,72$ \\
\hline$\Delta \mathrm{V}-\mathrm{N} 20$ calcule/Nt & 3,02 & 4,95 & 0,56 & 2,04 & 3,21 \\
\hline $\begin{array}{c}\Delta \mathrm{N}-\mathrm{N} 20 \text { mesure } / \mathrm{Nt} \\
\text { Juac (en } \% / \mathrm{h})\end{array}$ & 1,15 & 0,73 & 0,66 & 0.56 & 0,75 \\
\hline $\mathrm{AN}(\mathrm{NO} 3+\mathrm{NO} 2+\mathrm{NH} 4) / \mathrm{Nt}^{*}$ & 0,21 & 4,08 & $-0,03$ & $-0,39$ & 0.58 \\
\hline$\Delta$ N organique $\mathbf{N t}$ & -2.34 & 1,79 & $-1,32$ & $-2,98$ & $-0,28$ \\
\hline$\Delta \mathrm{N}-\mathrm{N} 2 \mathrm{O}$ calculéiNt & 2,12 & $-5,87$ & 1,35 & 3,37 & $-0,3$ \\
\hline AN-N2O mesurélNt & 062 & 0,41 & 0,42 & 0,25 & 0,13 \\
\hline
\end{tabular}

$\cdot \mathrm{Nt}=\mathrm{Norg}{ }^{2}+\mathrm{N}-\mathrm{NH}_{4}{ }^{+}+\mathrm{N}-\mathrm{NO}^{+}+\mathrm{N}-\mathrm{NO3}^{-}+\mathrm{N}-\mathrm{N} 2 \mathrm{O}$ 
Pendant le temps dt, la surface de sédiment ds assure une dénitrification à vitesse constante $\mathrm{K}$ ( $\mathrm{mg}$ de $\mathrm{NO}_{3}-/ \mathrm{m}^{2} / \mathrm{j}$ ). La quantité de $\mathrm{NO}_{3}{ }^{-}$dénitrifiée $D$ dans la tranche d'eau est :

$\mathrm{D}=\mathrm{K} * \mathrm{dS} * \mathrm{dt}$ c'est-à-dire $\mathrm{D}=\mathrm{K} * \mathrm{dl} * 1 * \mathrm{dt}$ avec $K$ en $\mathrm{mg} / \mathrm{m}^{2} / \mathrm{j}$, dl en $\mathrm{m}, 1$ en $\mathrm{m}$ et dt en $\mathrm{j}$.

D'autre part, la quantité de $\mathrm{NO}_{3}{ }^{-}$disparue dans la tranche d'eau peut aussi être exprimée en fonction des concentrations à $\mathrm{t}=0$ et $\mathrm{t}=\mathrm{dt}$ :

$D=\left[\left(C * p^{*} l^{*} d l\right)-\left((C+d C) * p^{*} 1 * d\right)\right] * 1000$ soit $\mathrm{D}=-\mathrm{dC} * \mathrm{dl} * \mathrm{p} * 1 * 1000$ (2)

avec $\mathrm{dC}$ en $\mathrm{mg} / \mathrm{l}$, dl, $\mathrm{p}$ et $\mathrm{l}$ en $\mathrm{m}$.

L'égalité des termes (1) et (2) conduit à : $-\mathrm{dC} * \mathrm{p} * 1000=\mathrm{K} * \mathrm{dt}$

soit $: \mathbf{d C} / d t=-K / 1000 *$ p avec dC/dt en $\mathrm{mg} / \mathrm{l} / \mathrm{j}$, $K$ en $\mathrm{mg} / \mathrm{m}^{2} / \mathrm{j}$ et $\mathrm{p}$ en $\mathrm{m}$.

Les valeurs mentionnées dans le tableau 6 représentent des moyennes sur la durée de l'expérimentation avec indication des écarts mini-maxi. Sur un cycle annuel et dans les limites des conditions environnementales mentionnées, il en ressort que le taux moyen de dénitrification dans la rivière Charente est de :

$28,44 \mathrm{mg}$ de $\mathrm{NO}_{2}-/ \mathrm{m}^{2} / \mathrm{h}$ soit un facteur $\mathrm{K}$ égal à $682 \mathrm{mg}$ de $\mathrm{NO}_{3}-/ \mathrm{j}$ ou encore $2,5 \mathrm{t} / \mathrm{ha} / \mathrm{an}$.

En prenant, pour le modèle, les valeurs références suivantes : $\mathrm{K}=682 \mathrm{mg} \mathrm{NO} 0_{3} / \mathrm{m}^{2} / \mathrm{j}$ et $\mathrm{p}=3 \mathrm{~m}$, on arrive à :

$$
\mathrm{dC} / \mathrm{dt}=-0,23 \mathrm{mg} / \mathrm{l} / \mathbf{j}
$$

Le facteur $K\left(154 \mathrm{mg}\right.$ de $\left.\mathrm{N} / \mathrm{m}^{2} / \mathrm{j}\right) \mathrm{s}^{\prime}$ insère dans la gamme de valeurs citées dans la littérature en ce qui concerne les études réalisées en rivière avec la méthode d'inhibition à l'acétylène (Tableau 7).
Tableau 6. Valeurs moyennes des principaux paramètres de la dénitrification.

Table 6. Average values of main denitrification parameters.

\begin{tabular}{|c|c|c|c|c|}
\hline & $\begin{array}{c}\mathrm{O} 2 \\
\mathrm{mg} / \mathrm{A}\end{array}$ & $\begin{array}{c}\text { Temperature } \\
{ }^{\circ} \mathrm{C}\end{array}$ & $\begin{array}{l}\mathrm{NO3} \\
\mathrm{mg} / 1\end{array}$ & $\begin{array}{c}\text { NO3 dénitrifıé } \\
\text { mg/m2/h } \\
=\text { Facteur } \mathbf{K}\end{array}$ \\
\hline NERSAC & $\begin{array}{c}6.0 \\
(0.2-11.5)\end{array}$ & $\begin{array}{c}16.4 \\
(9.5 \cdot 22,3)\end{array}$ & $\begin{array}{c}12.8 \\
(5.7-19.9)\end{array}$ & $\begin{array}{c}24.31 \\
(15-4 I)\end{array}$ \\
\hline JUAC & $\begin{array}{c}7.2 \\
(4,3+10,7)\end{array}$ & $\begin{array}{c}17.1 \\
(9.8 .23 .2)\end{array}$ & $\begin{array}{c}16.5 \\
(5.8 .23 .4)\end{array}$ & $\begin{array}{c}32.56 \\
(5.103)\end{array}$ \\
\hline Ensemble & 6.6 & 168 & 14.7 & 28.44 \\
\hline
\end{tabular}

\section{Conclusion}

La comparaison de l'estimation du taux de denitrification par dosage direct (méthode à l'acétylène) à celle fournic par l'analyse des formes de l'azote (défaut de bilan) montre la difficulté d'utiliser un déficit de bilan, méthode qui est donc à exclure. En moyenne, $0,6 \%$ (SD $\pm 0,3$ ) de l'azote total de nos enceintes est dénitrifié par heure, alors que le calcul théorique estime ce pourcentage à $1,4(\mathrm{SD} \pm 3)$.

Cette assez bonne concordance ne semble imputable qu'au hasard. En effet, la première estimation s'accompagne d'un coefficient de variation de $51 \%$. Par la méthode des bilans, il y a extrapolation des données non mesurées et l'estimation, dans ce cas, s'assortit d'un coefficient de variation bien plus élevé (206\%).

D'autre part, la méthode des bilans azotés ne prend en compte que les variations dans l'eau des

Tableau 7. Queiques valeurs de taux de dénitrification citees dans la littérature (d'après Seitzinger 1988 et Knowles 1982).

Table 7. Some denitrification rates in the literature.

\begin{tabular}{|c|c|c|}
\hline Localtsation & $\operatorname{Taux}\left(\mathrm{mg} N . m^{-2} \mathrm{j}^{-1}\right)$ & Reterence \\
\hline \multicolumn{3}{|l|}{ Rlvlères } \\
\hline San Francisquito Creck, Californic & 16 & Duff et al, 1984 \\
\hline Little Lost Man Creek & 0 & Duff et al, 1984 \\
\hline Seine, France & 240 & Chestérikoff et al., 1990 \\
\hline \multicolumn{3}{|r|}{ - } \\
\hline Hampen, Danemark & $1,6-16,8$ & Christensen et Sorensen, 1986 \\
\hline \multicolumn{3}{|l|}{ lac moderement eutrophe } \\
\hline \multicolumn{3}{|l|}{ Lacs d'eau douce } \\
\hline Canada, mésotrophe & $2.4-7.2$ & Chan et al, 1979 \\
\hline Gravières France & 0.336 & Helmer et Labroue, 1992 \\
\hline
\end{tabular}


cloches. Elle surestime la dénitrification en occultant les interactions de l'interface eau-sédiment où l'assimilation des nitrates concourt à la synthèse d'azote organique.

Par rapport à la méthode des bilans, le dosage direct par blocage à l'acétylène apparait plus précis tout en concervant encore une approche en $«$ boîte noire ": quel que soit le sens des échanges biochimiques et leur intensité, il quantifie un produit issu d'une activité enzymatique, passage obligé des nitrates vers l'azote moléculaire, et présent uniquement dans l'eau. La disparition vraie d'azote de la tranche d'eau est probablement supérieure à la dénitrification mesurée par la méthode à l'acétylène : en effet, l'acétylène, inhibant la nitrification, peut conduire, par le biais de son rôle sur la concentration en nitrate, à sous-estimer la dénitrification. De plus, les échanges eau-sédiment n'étant sans doute pas totalement réversibles, leur solde peut aboutir à une part de disparition d'azote par diagenèse.

Dans l'exemple de la Charente, il faut enfin souligner l'importance de ce phénomène de dénitrification avec au minimum 2,5 tonnes de nitrates éliminés annuellement par hectare de lit de rivière et. sa constance lorsque la matière organique dégradable et les nitrates sont régulièrement apportés par la pollution entrante (effluents urbains et industriels) et bien que les eaux soient toujours suffisamment oxygénées.

L'étude du rôle des macrophytes et du périphyton et l'influence de l'épaisseur de la tranche d'eau au-dessus du sédiment sur la dénitrification permettra d'affiner ce type de mesure in situ.

\section{Travaux cites}

Chan Y.K., Knowles R. 1979. - Measurement of denitrification in two freshwater sediments by an in situ acetylene inhibition method. Appl. Environ. Microbiol, 37 : 1067-1072.

Chestérik off A., Garban B. \& Ollivon D. 1990. Origine et dynamique de l'azote en Seine à l'aval de Paris. Comportement des MES, du phosphore et du COP : rôle de la station d'Achères dans cette problématique. Rapport technique Piren-Seine, $1 / 90 / 03$.

Christensen P.B. \& Sorensen J. 1986. - Temporal variation of denitrification activity in plant-çovered littoral sediment from lake Hampen, Denmark. Appl. Environ. Microbiol., 51 : 1174-1179.
Duff J.H., Triska F.J. \& Oremland R.S. 1984. -- Denitrification associated with stream periphyton : chamber estimates from undisrupted communities. J. Environ. Qual., 13 : 514-518.

Fradet E. 1989. - Dénitrification en rivière, relations avec les propriétés physicochimiques de l'eau et des sédiments. D.E.A., Laboratoire Biologie des Sols, Université ClaudeBernard, Lyon, 69 p.

Gorlenko V.M., Dubinina G.A.\& Kuznetsov S.I. 1983. - The ecology of aquatic micro-organisms. W. Ohle Ed. E. Schweizerbart'sche Verlagbuchhandlung (Nägele u. Obermiller), Stuttgart : $252 \mathrm{p}$.

Helmer C. \& Labroue L. 1992. - Denitrification in gravel-pit lakes. Hydrobiologia (sous presse).

Knowles R. 1982, - Denitrification. Microbiological Reviews, 46, $1: 43-70$.

Labroue L., Toureng J.N., Mieussens C., Robert J. \& Donville B. 1988. - Rôle des lacs de gravières dans la diminution des teneurs en nitrates des aquifères alluviaux de la vallée de la Garonne. Essai de quantification. Annls Limnol., 24 (1) : 31-38.

Labroue L., Delmas R., Serça D. \& Dagnac J. 1991. - La pollution nitratée des nappes phréatiques facteur de pollution atmosphérique. C.R. Acad. Sc., Paris, 313, III, 119-124.

Ma Z.C. 1991. - Modélisation du transfert des nitrates : du bassin de recherche a u grand bassin (exemples des bassins de

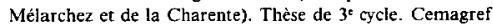
(groupement d'Antony. Division Hydrologie)/ENTRTS., Université Louis Pasteur, Strasbourg : $183 \mathrm{p}+$ annexes.

Messer J. \& Brezonik P.L. 1983. - Comparison of denitrification rate estimation techniques in a large, shallow lake. Wat. Res., $17: 631-640$.

Painter H.A. 1977. - Microbial transformations of inorganic nitrogen. Prog. Wat. Tech, 8, 4/5: 3-29.

Randall M.J. \& Ingraham J.L, 1986. - The denitrifying prokaryotes. In M.P. Starr Stolp, H., Trüper, H.G., Balows, A. Schlegel, H.G., The prokaryoles : a handbook on habitats, isolation, and identification of bacteria., Springer-Verlag Berlin Heidelberg, 1 : 913-925.

Seitzinger S.P. 1988. - Denitrification in freshwater and coastal marine ecosystems : ecological and geochemical signifiance. Limnol. Oceanogr. 33 (4/2) : 702-724.

Tiedje J.M., Sexstone A.J., Myrold D.D. \& Robinson J.A. 1982. - Derutrification : ecological niches, competition and survival. Antonie Van Leeuwenhoek, $48: 569-583$.

Tiedje J.M. 1988. - Ecology of denitrification and dissimilatory nitrate reduction to ammonium. in « Biology of ancerobic microorganisms ». A.J.B. Zehnder Ed. Wiley-Liss-NewYork: 179-244.

Toms I.P. 1975. - Factors affecting the removal of nitrate by sediments from rivers, lagoons and lakes Technical Report. Water Research Centre, 14: 23 p.

Whitehead P.\& O'Connelle. 1984. - Water quality modelling, forecasting and control. Proceedings of an international workshop at the Institute of Hydrology. Wallingford : Report 88. 